\title{
Prediction of Combustion Noise for an Aeroengine Combustor
}

\author{
Yu Liu* \\ University of Surrey, Guildford, GU2 7XH, United Kingdom \\ Ann P. Dowling ${ }^{\dagger}$ and Nedunchezhian Swaminathan ${ }^{\ddagger}$ \\ University of Cambridge, Cambridge, CB2 1PZ, United Kingdom
}

\begin{abstract}
Romuald Morvant\$, Michael A. Macquisten and Luca F. Caracciolo Rolls-Royce plc, Derby, DE24 8BJ, United Kingdom
\end{abstract}

Combustion noise may become an important noise source for lean-burn gas turbine engines, and this noise is usually associated with highly unsteady flames. This work aims to compute the broadband combustion noise spectrum for a realistic aeroengine combustor, and to compare with available measured noise data on a demonstrator aeroengine. A low-order linear network model is applied to a demonstrator engine combustor to obtain the transfer function that relates to unsteadiness in the rate of heat release, acoustic, entropic and vortical fluctuations. A spectral model is used for the heat release rate fluctuation, which is the source of the noise. The mean flow of the aeroengine combustor required as input data to this spectral model is obtained from RANS simulations. The computed acoustic field for a low-medium power setting indicates that the models used in this study capture the main characteristics of the broadband spectral shape of combustion noise. Reasonable agreement with the measured spectral level is achieved.

\footnotetext{
${ }^{*}$ Lecturer, Department of Mechanical Engineering Sciences, Member AIAA.

${ }^{\dagger}$ Professor, Department of Engineering, Trumpington Street, Fellow AIAA.

${ }^{\ddagger}$ Reader, Department of Engineering, Trumpington Street, Member AIAA.

$\S$ Acoustic Engineer, Noise Engineering, SINB-38, PO Box 31.

『 Aerothermal Engineer, Combustion Systems Engineering, SINA-76, PO Box 31.
} 


\section{Introduction}

Yombustion noise may become an important noise source if not well understood and $\checkmark$ controlled at the design stage of lean-burn gas turbine combustors because lean burning involves highly unsteady flames. Since lean burning has been identified as a potential way forward for environmentally friendly engines with low emission, this noise source needs to be studied. A priori knowledge of its source mechanisms is desirable and precise prediction methods are essential for design purposes. There are two components of combustion noise in gas turbines: the direct noise is due to pressure fluctuations generated directly by unsteadiness in the rate of heat released by combustion; and the indirect noise is produced by entropy fluctuations and also generated by fluctuations in the rate of heat release, which produce reflected acoustic waves as they accelerate through the turbine inlet guide vanes. Modeling of both direct and indirect broadband noise requires a sound understanding of the characteristics of the underlying heat release fluctuations that are ultimately the combustion noise source, and the transfer function that relates these acoustic and entropic fluctuations to the unsteady heat release rate. While previous work has extensively studied the sound emission from open flames (e.g. Refs. 1,2,3,4) without the effects of enclosures, the attention received to predict the spectral characteristics of combustion noise in the complicated configuration of a realistic aeroengine combustor is far from sufficient in literature.

In the present study, we employ modeling methods to compute the broadband spectrum of combustion noise, and aim to validate the models by comparing the calculated results with available measurement of combustion noise spectra on a demonstrator aeroengine. A schematic flowchart of the modeling method is illustrated in figure 1. For open flames in free space, an analytical solution exists for the pressure fluctuations generated by specified fluctuations in heat release rate, i.e. the transfer function. However for an aeroengine

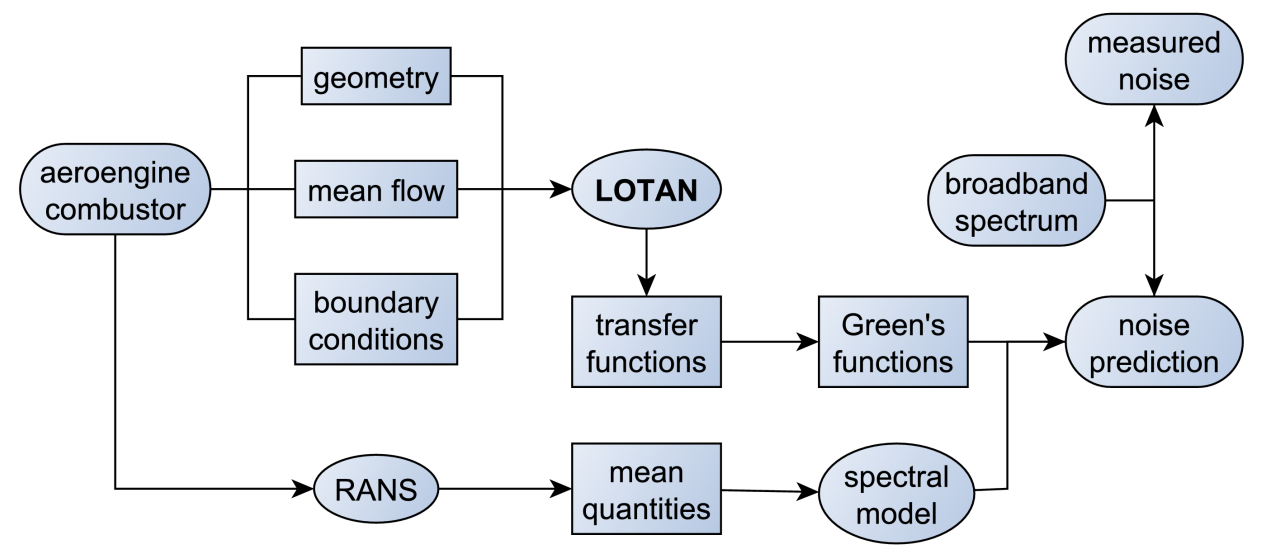

Figure 1. Schematic flowchart of the prediction model. 
combustor in an enclosed system, the transfer function needs to be calculated numerically since it varies spatially and temporally. This numerical computation is the primary objective of this paper, and it is achieved by using a computer program called LOTAN based on low-order linear acoustical network analysis.

A spectral model $^{5}$ for turbulent premixed flames is applied to represent the thermoacoustic sources. This model has been shown to be capable of providing quantitative acoustic spectra based on local mean turbulence and mean heat release rate. In the model, a local wave number model spectrum of heat release rate fluctuation is mapped to a frequency spectrum required to calculate the sound pressure spectrum. The spectral model was validated experimentally on premixed swirling jet flames ${ }^{5,6}$ with underpredictions observed at low frequencies. ${ }^{5,7,6}$ This deficiency is improved in this paper after applying corrections to the model. The input of mean quantities for this model can be obtained from either measurement ${ }^{5,6}$ or CFD simulation. ${ }^{7}$ In the work reported here, it is obtained from Reynolds-averaged Navier-Stokes (RANS) calculations for the combustor flow field.

The paper commences with a brief description of the theoretical model in Sec. II, including the calculation of the Green's function for thin annular ducts using LOTAN and an outline of the spectral model. ${ }^{5}$ Section III discusses the LOTAN results of the Green's function, including its spatial variation, spectral features, the effects of source position, the reflected sound from entropy waves accelerating through the combustor exit, and flow paths in combustor and outer annulus, for the demonstrator aeroengine. Following the modeling methods, the predicted noise spectra for the demonstrator aeroengine are compared in Sec. IV with the measured noise data. The conclusions of the study are then summarized in Sec. V.

\section{Theoretical Modeling}

\section{II.A. Combustion noise spectrum}

The geometry of an aeroengine combustor is complicated. The air from compressor exit is split. The main flow path is through a premixer, where the fuel is introduced, and the mixture enters an annular combustor. Secondary flows through inner and outer annuli enter the combustor through ports and perforated cooling rings, as shown in figure 2. The flows at compressor exit and turbine entry are choked, giving simple inlet and outlet boundary conditions and ensuring that linear fluctuations generated in the combustor do not depend on compressor or turbine properties. Marble and $\mathrm{Candel}^{8}$ derived the boundary conditions at a choked exit. This has been confirmed experimentally by Leyko et al. ${ }^{9}$ using results from Bake et $a l .^{10}$ and has been extended to circumferential waves and annular nozzles. ${ }^{11}$ The reflected waves created when an upstream-propagating acoustic wave approaches a choked inlet nozzle have been determined by Stow et al. ${ }^{11}$ 


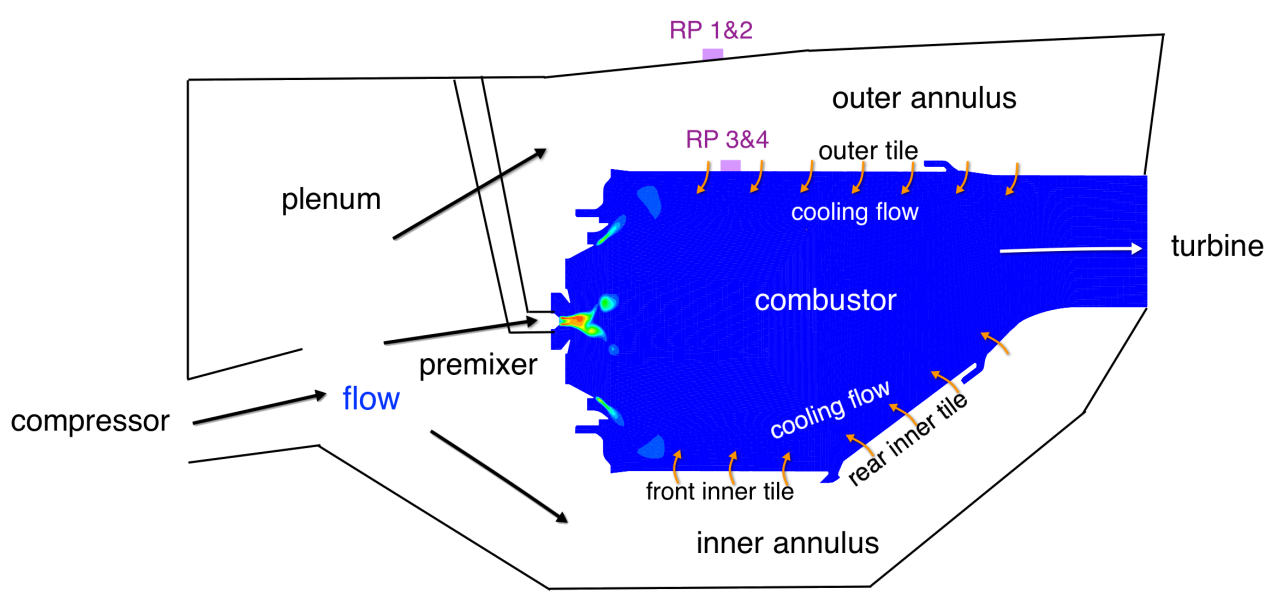

engine centerline

Figure 2. The schematic configuration of the demonstrator engine combustor.

We introduce the Green's function $\boldsymbol{G}(\boldsymbol{y}, \tau ; \boldsymbol{x}, t): G_{1}$ represents the pressure generated at $(\boldsymbol{x}, t)$ due to an impulse of rate of heat addition $\delta(\boldsymbol{x}-\boldsymbol{y}, t-\tau)$ at $(\boldsymbol{y}, \tau) ; G_{2}$ similarly describes the entropy produced and $G_{3}, G_{4}$ and $G_{5}$ the vorticity. $\boldsymbol{G}$ is the solution of the linearised Euler equations from compressor exit to turbine entry that satisfies the appropriate inlet and outlet boundary conditions. The determination of this Green's function will be discussed further in Sec. II.B.

The pressure perturbation $p^{\prime}(\boldsymbol{x}, t)$ generated by a rate of heat input $\dot{q}^{\prime}(\boldsymbol{y}, \tau)$ per unit volume is then given by

$$
p^{\prime}(\boldsymbol{x}, t)=\int_{-\infty}^{\infty} \int_{v_{f}} G_{1}(\boldsymbol{y}, \boldsymbol{x}, t-\tau) \dot{q}^{\prime}(\boldsymbol{y}, \tau) \mathrm{d}^{3} \boldsymbol{y} \mathrm{d} \tau
$$

where $v_{f}$ denotes the volume of the flame brush and the prime denotes a perturbation from the mean. A similar integral can be written for the entropy fluctuation at $(\boldsymbol{x}, t)$ using $G_{2}(\boldsymbol{y}, \tau ; \boldsymbol{x}, t)$. Following the Fourier transform of Eq. (1) one obtains

$$
\hat{p}(\boldsymbol{x}, \omega)=\int_{v_{f}} \hat{G}_{1}(\boldsymbol{y}, \boldsymbol{x}, \omega) \hat{\dot{q}}(\boldsymbol{y}, \omega) \mathrm{d}^{3} \boldsymbol{y},
$$

where $\omega$ is the angular frequency.

The power spectral density $\hat{P}(\boldsymbol{x}, \omega)$, a measurable quantity in experiments, can be simply obtained as

$$
\hat{P}(\boldsymbol{x}, \omega)=\int_{v_{f}} \int_{v_{f}} \overline{\hat{G}_{1}\left(\boldsymbol{y}_{a}, \boldsymbol{x}, \omega\right) \hat{G}_{1}^{*}\left(\boldsymbol{y}_{b}, \boldsymbol{x}, \omega\right)} \cdot \psi_{q}\left(\boldsymbol{y}_{a}, \boldsymbol{y}_{b}, \omega\right) \mathrm{d}^{3} \boldsymbol{y}_{a} \mathrm{~d}^{3} \boldsymbol{y}_{b},
$$


where $\psi_{q}$ is the power spectral density of $\dot{q}^{\prime}$,

$$
\psi_{q}\left(\boldsymbol{y}_{a}, \boldsymbol{y}_{b}, \omega\right)=\frac{1}{2 \pi} \int_{-\infty}^{\infty} \overline{\hat{\dot{q}}\left(\boldsymbol{y}_{a}, \omega\right) \hat{\dot{q}}^{*}\left(\boldsymbol{y}_{b}, \omega\right)} \mathrm{d} \omega
$$

the overline denotes an averaging process, the asterisk indicates the complex conjugate, and $\boldsymbol{y}_{a}, \boldsymbol{y}_{b}$ are two locations within the flame brush $v_{f}$. It is assumed that the Green's function has negligible variation over the correlation volume for the fluctuating heat release rate, which will be confirmed in Sec. III.D. The correlation spectrum of the heat release rate, $\psi_{q}\left(\boldsymbol{y}_{a}, \boldsymbol{y}_{b}, \omega\right)$, needs to be integrated over the flame brush $v_{f}$. As shown in Wäsle ${ }^{12}$ this amounts to integrating the contributions from the coherent monopole sources of combustion noise with a correlation volume $V_{\text {cor }}$ over the flame volume. Therefore the power spectral density in Eq. (3) is reduced to

$$
\hat{P}(\boldsymbol{x}, \omega) \approx \int_{v_{f}}\left|\hat{G}_{1}(\boldsymbol{y}, \boldsymbol{x}, \omega)\right|^{2} \cdot \psi_{q}(\boldsymbol{y}, \omega) V_{\text {cor }} \mathrm{d}^{3} \boldsymbol{y}
$$

The sound pressure level (SPL) is hence characterized by $\hat{P}(\boldsymbol{x}, \omega)$ as $10 \log _{10}\left(\hat{P} / p_{\text {ref }}^{2}\right)$, where the reference acoustic pressure is $p_{\text {ref }}=2 \times 10^{-5} \mathrm{~Pa}$.

In Secs. II.B and III we discuss the calculation and results of the Green's function and in Sec. II.C models for $\psi_{q}$ and $V_{\text {cor }}$ are summarised. These are combined in Sec. IV to predict the noise spectrum in an aeroengine combustor.

\section{II.B. Green's function for thin annular ducts}

The Green's function determines the linear acoustic, entropic and vortical waves due to a harmonic variation in the heat release rate. We calculate its Fourier transform $\hat{\boldsymbol{G}}(\boldsymbol{y}, \boldsymbol{x}, \omega)$ using a computer program LOTAN which was developed from a low-order thermoacoustic network model to predict combustion oscillations in lean premixed prevaporized (LPP) gas turbine combustors. $\hat{\boldsymbol{G}}(\boldsymbol{y}, \boldsymbol{x}, \omega) e^{i \omega t}$ determines the waves at $\boldsymbol{x}$ due to a harmonically varying spatially concentrated rate of heat input $\delta(\boldsymbol{x}-\boldsymbol{y}) e^{i \omega t}$. The program is intended for thin annular combustors, typical of aeroengines, so axial and circumferential variations are included but radial dependence is ignored. An introduction to the theory behind the LOTAN program can be found in Stow. ${ }^{13,14,15}$

In a LOTAN calculation the geometry is constructed as a series of modules with jump conditions between them. ${ }^{16}$ The linear waves propagate or convect through straight portions of the geometry. The appropriate conservation conditions are applied across the combustion zone, any changes in cross-sectional area and where flow paths split or join. Together with the inlet and outlet boundary conditions this leads directly to the modal transfer functions, 
for example $(\hat{p} / \hat{q})_{n}$, in circumferential mode number $n$. By using the Green's function technique by Hedge et al. ${ }^{17}$ and the routine method of eigenfunctions to solve the Green's function, ${ }^{18}$ the pressure response to a concentrated source $\frac{1}{r} \delta\left(\theta-\theta_{0}\right) \delta\left(r-r_{0}\right) \delta\left(x-x_{0}\right) e^{i \omega t}$ at circumferential angle $\theta_{0}$ can be expressed as a superposition of circumferential modes of the transfer function, i.e.

$$
\hat{G}_{1}(\boldsymbol{y}, \boldsymbol{x}, \omega)=\sum_{n=-N}^{N}(\hat{p} / \hat{\dot{q}})_{n} e^{i n\left(\theta-\theta_{0}\right)}
$$

as shown in figure 1. Note that since the summation over circumferential modes is theoretically infinite, we should take $N$ to be sufficiently large so that the truncation error is small.

The unsteady heat input can generate entropic and vortical waves as well as acoustic wave. These are coupled at the choked combustor exit where incident entropy and vortical disturbances lead to reflected pressure waves which need to be included in combustion noise calculations. If one ignores the reflected pressure waves, then the resulting noise is the direct noise. The total combustion noise (direct + indirect) is obtained by including all the contributions. The differences in the spectrum between the direct and full combustion noise for a gas turbine combustor are discussed in Sec. III.D.

The LOTAN program can calculate the frequency spectrum and mode shape of the transfer functions for an observer at $\boldsymbol{x}(r, \theta, x)$ and the source at $\boldsymbol{y}\left(r_{0}, \theta_{0}, x_{0}\right)$. By running LOTAN for different axial source position $x_{0}$ and varying the values of $\theta_{0}$ in Eq. (6), one obtains the dependence of $\hat{G}_{1}(\boldsymbol{y}, \boldsymbol{x}, \omega)$ on the source position. Because the mean flow is axisymmetric, the transfer function for a negative mode is equivalent to the mode of the corresponding positive value. Summing these two contributions from the $\pm n$ modes gives the Green's function in Eq. (6) as

$$
\hat{G}_{1}(\boldsymbol{y}, \boldsymbol{x}, \omega)=(\hat{p} / \hat{\dot{q}})_{n=0}+2 \sum_{n=1}^{N}(\hat{p} / \hat{\dot{q}})_{n} \cos \left[n\left(\theta-\theta_{0}\right)\right]
$$

which means that only positive mode numbers need to be computed thereby nearly halving the computational time required to obtain $\hat{G}_{1}(\boldsymbol{y}, \boldsymbol{x}, \omega)$ using LOTAN.

\section{II.C. Spectral model for heat release rate}

A model for the heat release rate spectrum $\psi_{q}(\boldsymbol{y}, \omega)$ in Eq. (5) is obtained as follows. The use of a reaction progress variable $c$ taking a value of 0 in the unburnt and 1 in the fully bunt mixtures, in turbulent premixed combustion calculations is common. Since the mixture is homogeneously mixed in premixed combustion, the fluctuations in $c$ must result from 
fluctuations in reaction (or heat release) rate. Thus, one can write

$$
\dot{q}^{\prime}(\boldsymbol{y}, t)=\mathcal{A} c^{\prime}(\boldsymbol{y}, t)
$$

where $\mathcal{A}$ is a constant of order unity to be determined later. It is straightforward to write the spectrum as $E_{q}(\kappa)=\mathcal{A}^{2} E_{c}(\kappa)$, where $\kappa$ is the wave number, by taking the Fourier transform on both sides of the above expression. If one integrates this expression over all wave numbers then $\overline{\dot{q}^{\prime 2}}=\mathcal{A}^{2} \overline{c^{\prime 2}}$. Since the reaction rate is a highly intermittent signal, its root mean square (rms) value can be as high as its mean value, ${ }^{4}$ and thus $\overline{\dot{q}}=\mathcal{A} \sqrt{\overline{c^{\prime 2}}}$. A similar expression has been obtained by Spalding ${ }^{19}$ for turbulent premixed combustion at sufficiently large turbulence Reynolds number $R e_{T}=u^{\prime} \Lambda / a_{0}$ with the turbulence intensity $u^{\prime}$ and integral length scale $\Lambda$ larger than the laminar flame speed $s_{L}$ and laminar flame thickness $\delta_{L}$.

It is well known ${ }^{20}$ that the presence of the rms of $c$ leads to a singular behaviour for the mean heat release rate, $\overline{\dot{q}}$ when the flamebrush leading or trailing edge is approached, that is when the mean value of $c$ going towards 0 or 1 , and this is avoided in practical simulations by replacing the rms value by the variance. ${ }^{20}$ This gives a model for the mean heat release rate $\operatorname{as}^{21}$

$$
\overline{\dot{q}}=\rho_{0} Y_{f, 0} H \widetilde{\xi}=\rho_{0} Y_{f, 0} H C_{D}(\widetilde{\epsilon} / \widetilde{k}) \widetilde{c^{\prime 2}}
$$

where $\widetilde{\xi}$ is the mean scalar dissipation rate of the progress variable variance $\widetilde{c^{\prime 2}}$, and $C_{D}=2.0$ is a model constant as proposed in Peters. ${ }^{21}$ The symbols $\rho_{0}, Y_{f, 0}$ and $H$ are the reactant density, fuel mass fraction and lower heating value of the fuel respectively. The dissipation rate of turbulence kinetic energy, $\widetilde{k}=1.5 u^{\prime 2}$, is $\widetilde{\epsilon}=u^{\prime 3} / \Lambda$. This mean heat release rate model then identifies

$$
\mathcal{A}=\rho_{0} Y_{f, 0} H C_{D}(\widetilde{\epsilon} / \widetilde{k})
$$

While other models proposed recently for $\widetilde{\xi}$ (see Ref. 22 for a summary of these models) are available, the above model is chosen for its simplicity and to limit the number of model parameters in the spectral description. Furthermore, these recent models also suggest the balance between the production and dissipation of progress variable fluctuation in a broad sense, including contributions coming from thermochemical processes. Nevertheless, it is worth to explore these models in a future study.

The wave number spectrum of the heat release rate, $E_{q}(\kappa)$, then becomes

$$
E_{q}(\kappa)=\left(\rho_{0} Y_{f, 0} H\right)^{2} C_{D}^{2}(\widetilde{\epsilon} / \widetilde{k})^{2} E_{c}(\kappa)
$$

where a correction has been made to the expression of $E_{q}$ in a similar approach used in an 
earlier study ${ }^{5}$ to account for the dimension of $E_{q}$. As has been done in that study ${ }^{5}$ one may use the model scalar spectrum derived by Tennekes and Lumley ${ }^{23}$ with their proposed constants $\alpha=1.5, \beta=0.3$ for $E_{c}$ if $c$ is presumed to be a passive scalar. The validity of this assumption requires a comprehensive analysis using laser diagnostics and direct numerical simulation data which is beyond the scope of this investigation. However, the spectrum of a reactive scalar reported in a recent study ${ }^{24}$ offers a reasonable support for the above assumption. After some algebraic rearrangement, the heat release spectrum $E_{q}$ is obtained as

$$
E_{q}(\kappa)=\rho_{0} Y_{f, 0} H \overline{\dot{q}} \cdot C_{D}^{2} C_{s} \widetilde{\epsilon}^{5 / 3} \widetilde{k}^{-2} \kappa^{-5 / 3} \cdot \exp \left[-\frac{3}{2}\left(\pi \beta \alpha^{1 / 2}(\kappa \Lambda)^{-4 / 3}+\alpha\left(\kappa \eta_{c^{2}}\right)^{4 / 3}\right)\right]
$$

where $C_{s}$ is an amplitude scaling function to be obtained below, and the Corrsin/Kolmogorov scale $\eta_{c}$ becomes $L_{C}=\left(a_{0}^{3} / \widetilde{\epsilon}\right)^{0.25}$ for a passive scalar. Since $c$ is a reactive scalar, Hirsch et $a l .{ }^{5}$ considered two effects:

1. The propagation speed of the flame front characterized by the Gibson scale $L_{G}=s_{L}^{3} / \widetilde{\epsilon}$ which will tend to "iron out" the small scales, i.e. produce a spectral cut off at high wave numbers. This effect is considered through an effective Corrsin length scale:

$$
\eta_{c}=\max \left[c_{G} L_{G}, L_{C}\right] \quad \text { with } \quad c_{G}=3 .
$$

2. To account for a change of combustion regime, the mean heat release rate model by Schmid et $a l .{ }^{25}$ is used as it explicitly includes the Damköhler number $D a$,

$$
\begin{aligned}
\overline{\dot{q}} & =4.96 \frac{\epsilon}{k}\left[s_{L}(2 / 3 k)^{-0.5}+\left(1+D a^{-2}\right)^{-0.25}\right]^{2} \cdot \tilde{c}(1-\tilde{c}) \rho_{0} Y_{f, 0} H, \\
D a & =\frac{0.09 k s_{L}^{2}}{\epsilon C_{c}^{2} a_{0}}
\end{aligned}
$$

where the constant $C_{c} \approx 1.2$ suggested for the natural gas fuel is employed in this work for the kerosene fuel used in the aeroengine combustor. The amplitude scaling function $C_{s}$ is derived as

$$
C_{s}=\frac{\alpha}{C_{D}} \frac{\widetilde{c^{\prime 2}}(D a)}{\widetilde{c^{\prime 2}}(D a \rightarrow \infty)}=\frac{\alpha}{C_{D}}\left[\frac{s_{L}(2 / 3 k)^{-0.5}+\left(1+D a^{-2}\right)^{-0.25}}{s_{L}(2 / 3 k)^{-0.5}+1}\right]^{2}
$$

In the limit of $D a \rightarrow \infty, C_{s}$ becomes $\alpha / C_{D}$

By combining Eqs. (12-16) one recognises that the local wave number spectrum for the heat release rate, $E_{q}(\kappa)$, is determined by the mean quantities. To obtain the frequency spectrum $\psi_{q}(\omega)$ required for the acoustic power spectrum, Hirsch et al. ${ }^{5}$ followed Tennekes 
and Lumley ${ }^{23}$ by requiring that the spectral energy content in the corresponding scales is conserved, i.e. $\kappa E_{q}=\omega \psi_{q}$, and postulating that the frequency spectrum is a simple rearrangement of the wave number spectrum, as has been used by Clavin \& Siggia. ${ }^{26}$ This leads to a mapping between wave number and frequency, strictly when homogeneity is assumed, which is reasonable for small scales relevant for combustion despite the large scales are inhomogeneous in turbulent premixed combustion. This mapping is written as

$$
\kappa=\frac{2 \pi}{\Lambda} \frac{2 \pi^{0.5}}{\alpha^{0.75}}\left(f \tau_{c}\right)^{1.5}
$$

where the characteristic time scale $\tau_{c}$ is given by

$$
\tau_{c}=C_{\tau} \frac{\delta_{T}}{s_{T}}=C_{\tau} \frac{\Lambda}{u^{\prime}} \frac{a_{0} /\left(s_{L} \Lambda\right)+\left(1+D a^{-2}\right)^{0.25}}{s_{L} / u^{\prime}+\left(1+D a^{-2}\right)^{-0.25}}
$$

using the turbulent burning velocity relations proposed by Schmid $^{27}$ with the model constant $C_{\tau} \approx 0.5$.

Now the local frequency spectrum of the heat release rate $\psi_{q}(\omega)=\kappa E_{q}(\kappa) / \omega$ is obtained by combining Eqs. (12-18). Moreover, it has been suggested in previous studies ${ }^{12,28}$ that the correlation volume is $V_{\text {cor }}=8 \ell_{\text {cor }}^{3}$ over the flame brush and $\ell_{\text {cor }} \approx \delta_{T}$, the flame brush thickness. Substituting these models for $\psi_{q}$ and $V_{\text {cor }}$ into Eq. (5), one obtains the combustion noise spectrum for thin annular combustors as

$$
\hat{P}(\boldsymbol{x}, \omega)=\frac{8}{\omega} \int_{v_{f}}\left|\hat{G}_{1}(\boldsymbol{y}, \boldsymbol{x}, \omega)\right|^{2} \cdot \kappa E_{q}(\kappa) \delta_{T}^{3} \quad \mathrm{~d}^{3} \boldsymbol{y}
$$

\section{The Green's Function for a Demonstrator Engine}

\section{III.A. The demonstrator engine combustor}

Measurements of the combustion noise spectrum for a demonstrator aeroengine ${ }^{\text {a }}$ have been conducted using pressure transducers ("rumble probes") in combustor and jet pipe. From the perspective of modeling the geometry within a LOTAN application, the demonstrator engine combustor consists of a diffuser plenum, 16 premixer ducts and the combustor, as illustrated in figure 2. The premixer ducts supply fuel and air to the combustor through the main flow path. Air also enters through perforated front and rear tiles. The annular combustor is surrounded by inner and outer annuli which supply cooling air to the combustor through outer and inner perforated tiles. There are thus six flow paths, i.e. the main flow (acoustic) path plus the paths in the outer annulus, inner annulus, outer tile, front and rear

\footnotetext{
${ }^{a}$ Rolls-Royce proprietary.
} 
inner tiles. Apart from this, fuel is also introduced via a pilot to ignite the main burner from the established pilot flame. The operating condition chosen for evaluation is at a low-medium power setting of $44 \%$ of maximum power (1747.5 rpm), where there is negligible fuel supplied to the pilot flame and the burning is in premixed mode.

A Rolls-Royce in-house code FLOWNET is used to generate the geometry and mean flow parameters as inputs for the LOTAN program (see figure 1). The geometry is composed of a network of modules which describe its features. Each flow path is modeled as a string of consecutive straight ducts with different cross-sectional areas and/or mean radii to account for the axially varying geometry. Appropriate conservation condition are applied across the junctions of ducts and zones of heat input. ${ }^{16}$ The premixer is modeled as a ring of ducts uniformly positioned circumferentially. Since the cross-stream dimension of a premix duct is small compared with the wavelength the flow within it is one-dimension (1-D). The cooling flow is considered by modeling the flow within the inner and outer annuli to be connected through a distribution of holes to the combustor flow. The input parameters for the mean flow are the pressure, axial velocity, density and temperature in each duct.

The boundary conditions on the mean flow are prescribed both at the inlet for $p, T$ and at the outlet for mass flow rate $\dot{m}$. Figure 3 shows the axial variations of pressure, axial
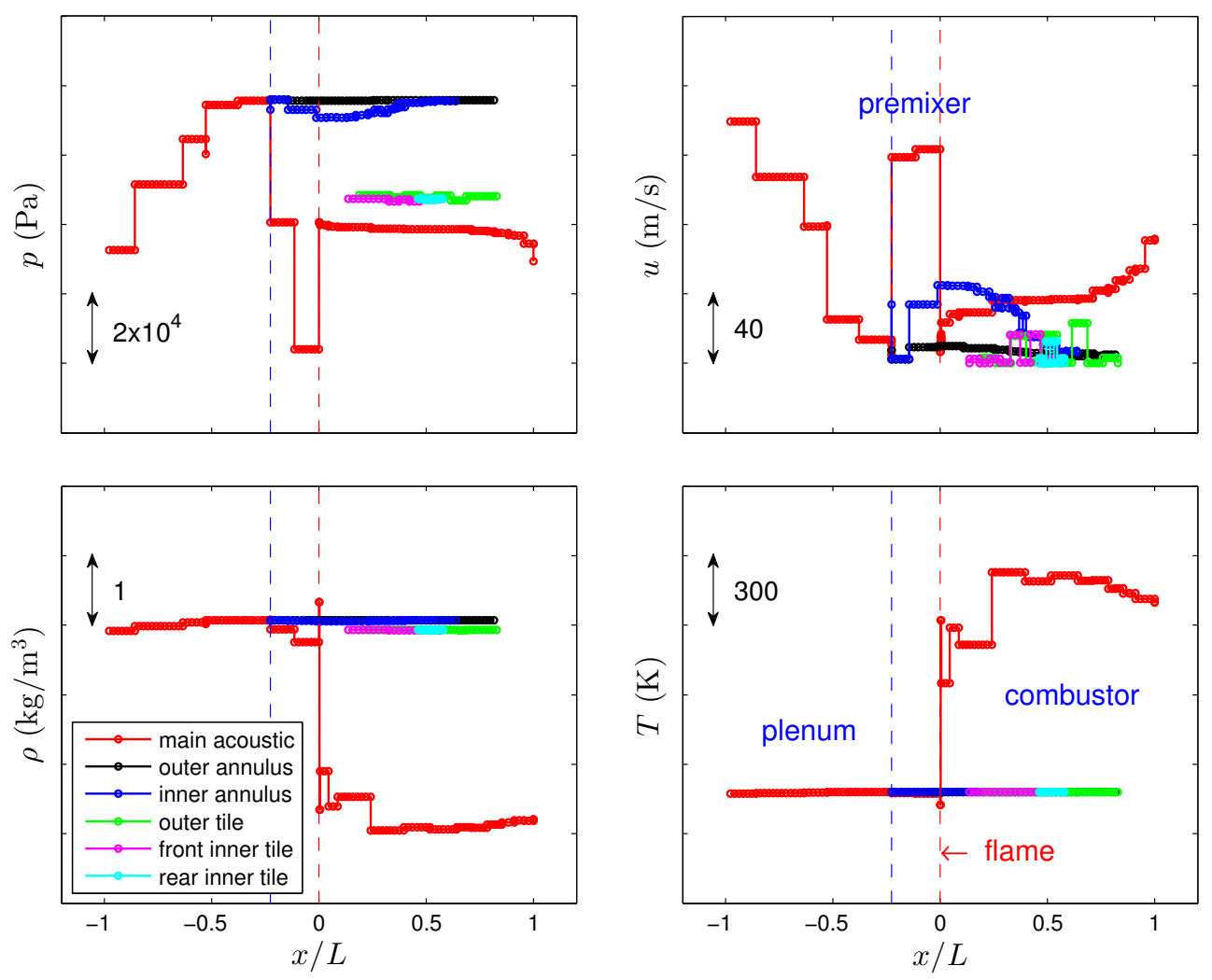

Figure 3. The axial variations of mean flow variables $p, u, \rho, T$ along all flow paths of the demonstrator engine. 
velocity, density and temperature for the mean flow along the various flow paths in the test geometry. The combustor inlet is located at $x / L=0.0$ and the axial coordinate is normalized using the combustor length $L$. As shown in Fig. 3, abrupt changes in the mean variables occur due to the geometry changes in the flow paths. In the main acoustic path, there is an evident jump in mean temperature at the beginning of the flame very close to the combustor inlet, and the drops in $T$ within the combustor are due to the cooling flow from other paths.

The boundary conditions applied are those of a choked inlet and outlet, representing approximations for the compressor outlet and turbine inlet flows respectively. The LOTAN code uses the choked boundary conditions described in Stow et al. ${ }^{11}$ for the inlet and outlet. The linear flow perturbations are calculated by propagating acoustic, entropic and vortical disturbances in straight ducts and annuli and joining ducts of different cross-sectional areas with appropriate conservation conditions. ${ }^{16}$

\section{III.B. Spatial variation}

The Green's function is obtained for the demonstrator engine as per Eq. (7). A restriction of LOTAN for modeling the junction of the 1-D premix ducts to the annular geometries of the plenum and combustor requires that the largest circumferential mode number must be less than half the number of the 1-D premixer ducts (16 in total for the demonstrator engine), and hence $N=7$ is used in Eq. (7). This has been checked to be sufficient to ensure small truncation errors as discussed in Sec. III.C.

Figure 4 shows the axial variations of the pressure-like Green's function $\left|\hat{G}_{1}(\boldsymbol{y}, \boldsymbol{x}, \omega)\right|$ in the six flow paths of the combustor at $100 \mathrm{~Hz}$ and $300 \mathrm{~Hz}$. At a low frequency of $100 \mathrm{~Hz}$ the
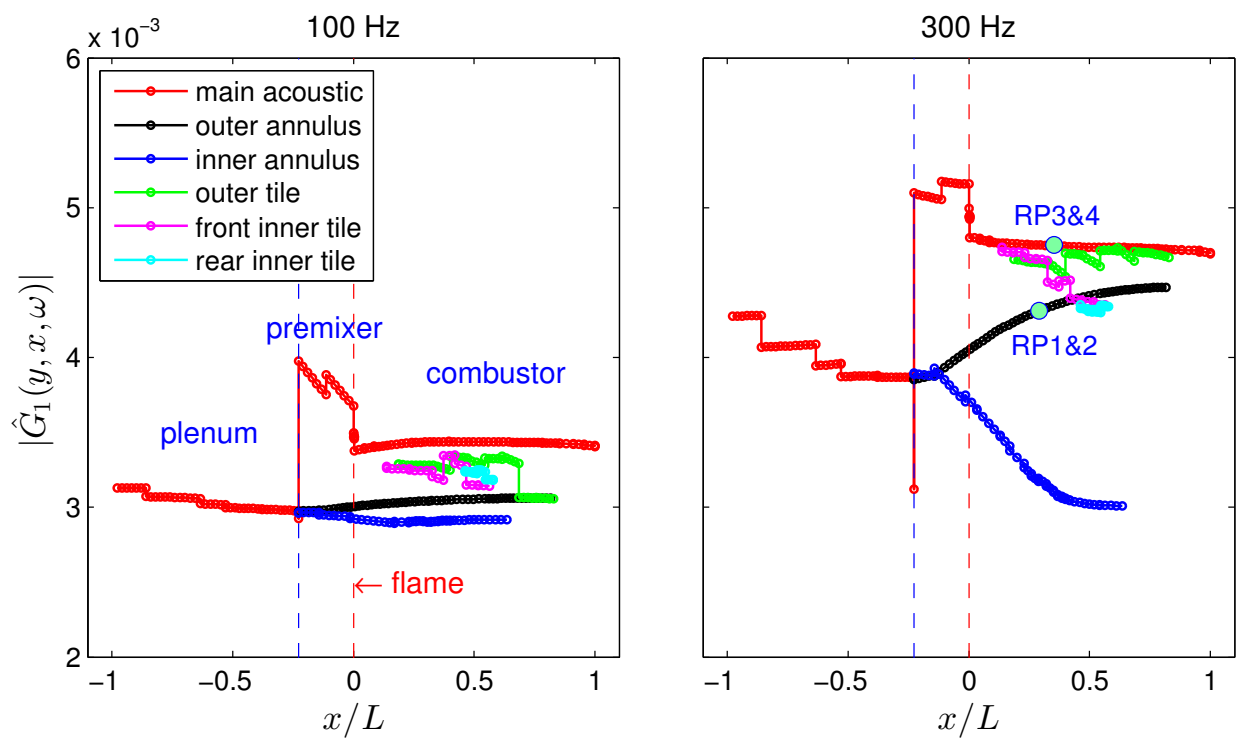

Figure 4. The axial variations of the Green's function $\left|\hat{G}_{1}(\boldsymbol{y}, \boldsymbol{x}, \omega)\right|$ in all flow paths of the demonstrator engine. $N=7, \theta=\pi / 3, \theta_{0}=0, x_{0}$ at combustor inlet. 
Green's functions shows very little variation in the amplitude of the pressure perturbation throughout the combustor apart from within the premixer ducts, where because of their smaller total cross-sectional area inertial effects are more important. At $300 \mathrm{~Hz}$ differences in amplitude become evident, particularly between $\left|\hat{G}_{1}\right|$ in the outer and inner annuli. There is little variation of $\left|\hat{G}_{1}\right|$ within the combustor $(0 \leqslant x / L \leqslant 1)$ for both frequencies. The noise measurements were performed using two pairs of "rumble probes", RP 1 \& 2 in the outer annulus and RP $3 \& 4$ in the combustor which is part of the main acoustic path. The axial positions of the rumble probes, as marked in the right graph in figure 4, are 0.292 (RP 1 \& 2) and 0.353 (RP $3 \& 4$ ) respectively, both of which are in the first half of the combustor.

The contours of $\left|\hat{G}_{1}(\boldsymbol{y}, \boldsymbol{x}, \omega)\right|$ in the $(x, \theta)$ plane for the frequencies 100, 300, 500 and $1000 \mathrm{~Hz}$ are shown in figure 5 to explore the spatial distribution of the Green's function within the combustor, which is the main acoustic path and is of most interest. As expected the Green's function is distributed symmetrically with respect to the source position $\theta_{0}=0$. $\left|\hat{G}_{1}\right|$ varies gradually in the circumferential direction $\theta$; again there is very little axial variation of $\left|\hat{G}_{1}\right|$ away from the source particularly in the region $\theta-\theta_{0} \neq 0$.
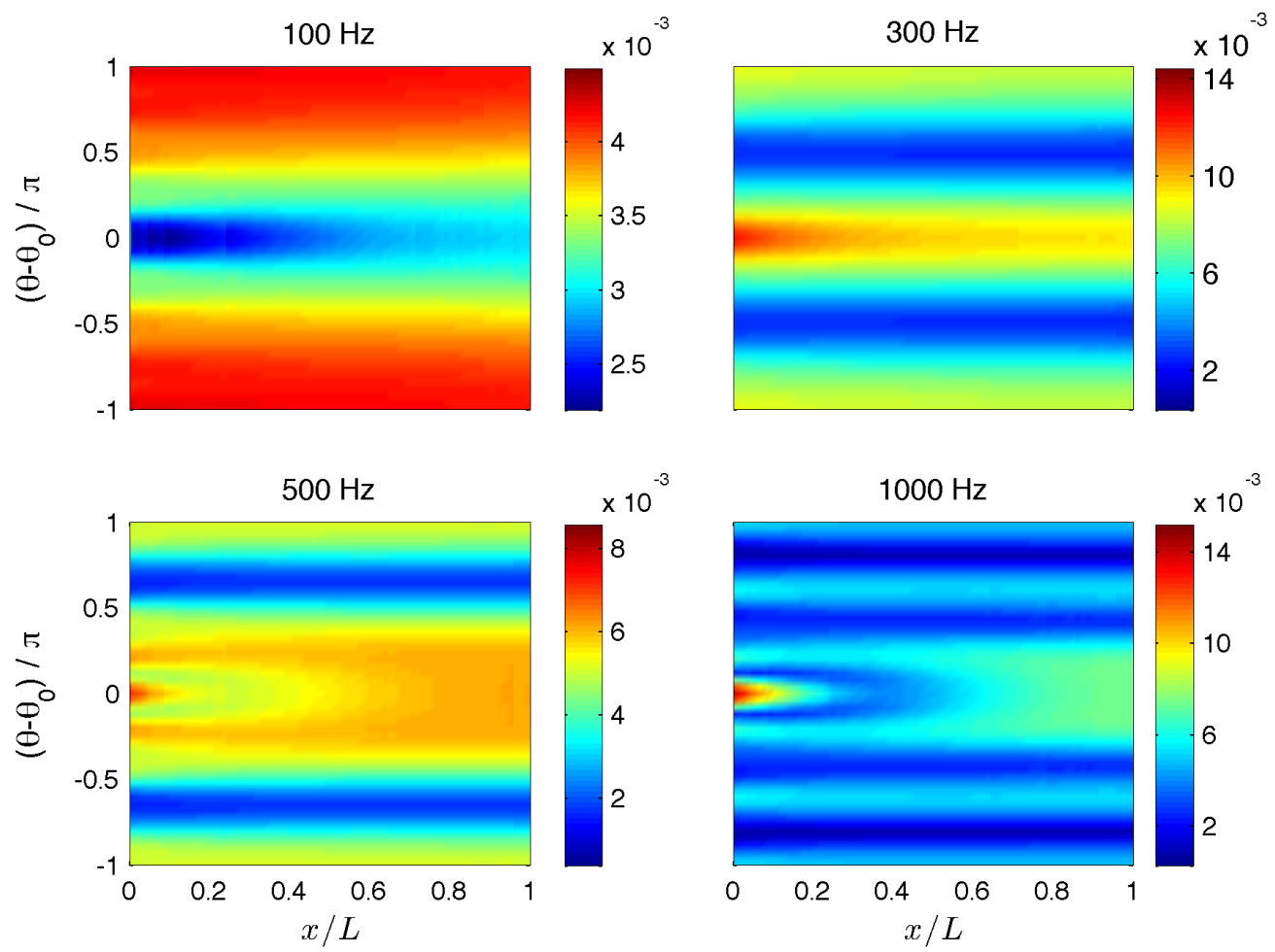

Figure 5. Contours of the Green's function $\left|\hat{G}_{1}(\boldsymbol{y}, \boldsymbol{x}, \omega)\right|$ on the $(x, \theta)$ plane in the main acoustic path. $N=7$, $\theta_{0}=0, x_{0}$ at combustor inlet. 


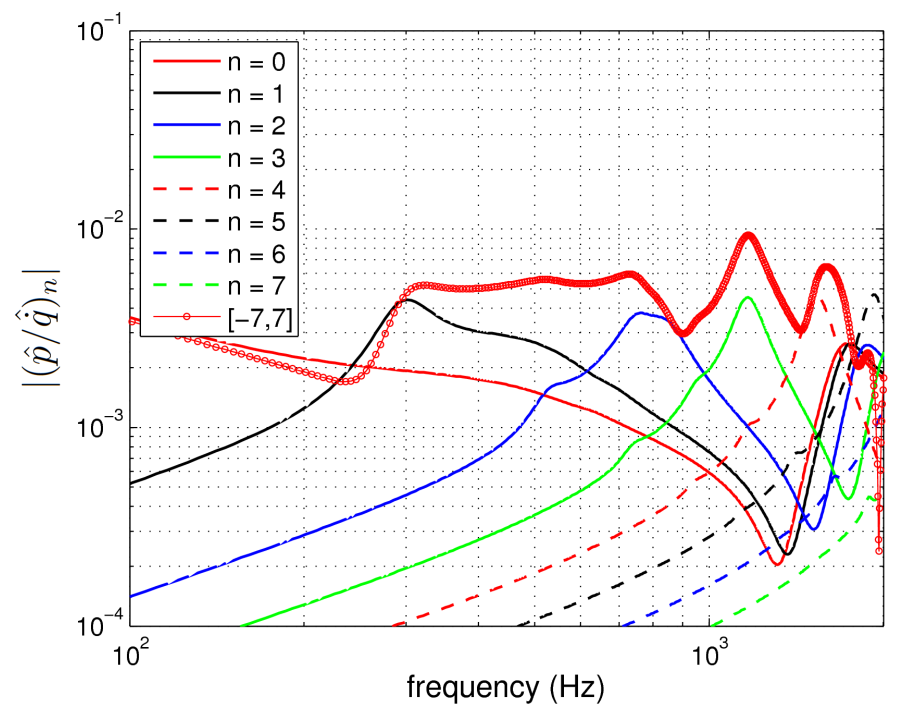

Figure 6. The spectra of $\left|(\hat{p} / \hat{\dot{q}})_{n}\right|$ of individual circumferential modes $0-7$ and the spectrum of the Green's function $\left|\hat{G}_{1}(\boldsymbol{y}, \boldsymbol{x}, \omega)\right| . N=7, \theta=\pi / 3, \theta_{0}=0 . x$ at RP $\mathbf{3} \& \mathbf{4}, x_{0}$ at combustor inlet.

\section{III.C. Spectra of individual modes and $G_{1}$}

To find out the relative contribution from each individual mode to the Green's function, the spectra of $\left|(\hat{p} / \hat{\dot{q}})_{n}\right|$ for the circumferential modes $n=0-7$ are plotted in figure 6 together with the spectrum of the Green's function by adding up the modes in $[-7,7]$. It is observed that the contributions from high-order modes to $\hat{G}_{1}$ are an order of magnitude smaller than those from low-order modes for low frequencies $(<1000 \mathrm{~Hz})$, and that the contribution from even higher modes is negligible. Therefore summing up to $N=7$ is sufficient to ensure accuracy of $\hat{G}_{1}$ for the demonstrator engine.

\section{III.D. Effects of source position and entropy wave}

The effect of source position on the Green's function has been investigated. If $\hat{G}_{1}$ is strongly dependent on $\boldsymbol{y}$, one needs to run LOTAN for a number of axial source positions $x_{0}$. In a lean-burn system, most of the heat release occurs within the first $15-20 \%$ of the combustor length. We consider the variation of $\hat{G}_{1}$ for a distribution of axial source positions within the first $24 \%$ of the combustor length. The actual values of $x_{0}$ for four representative source

Table 1. Axial positions of the selected source representing a fluctuation in heat release rate and the distances of the source to the combustor inlet.

\begin{tabular}{ccccc}
\hline \hline module no. & DG_B072 & DG_B080 & DG_B084 & DG_B088 \\
\hline$x_{0} / L$ & 0.002 & 0.086 & 0.158 & 0.240 \\
\hline \hline
\end{tabular}




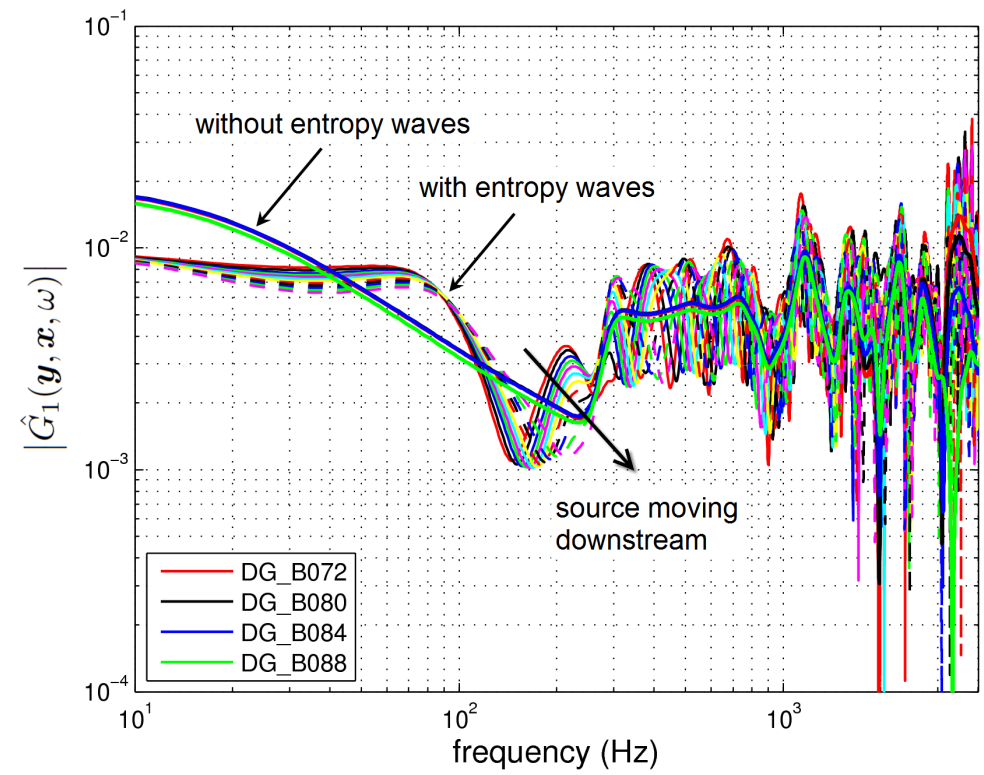

Figure 7. Effects of source position and entropy waves on spectrum of the Green's function $\left|\hat{G}_{1}(\boldsymbol{y}, \boldsymbol{x}, \omega)\right| . N=7$, $\theta=\pi / 3, \theta_{0}=0 . x$ at $\mathbf{R P} 3 \& 4$.

positions are summarized in table 1. Figure 7 shows the spectra of $\left|\hat{G}_{1}(\boldsymbol{y}, \boldsymbol{x}, \omega)\right|$ for these different source positions with (dashed lines) and without (solid lines) entropy waves incident of the exit nozzle. It confirms that $\left|\hat{G}_{1}\right|$ is not sensitive to the axial source position if entropy waves are assumed to have diffused before the combustor exit, particularly at low frequencies. This implies that when applying the prediction model in Eq. (19) it is safe to take $\hat{G}_{1}$ outside the source integral with respect to $x_{0}$, and hence that LOTAN needs to be run only once for each mode number.

The inclusion of entropy waves at the combustor exit, however, produces a frequency variation on top of the underlying trend in spectral levels as in figure 7. The variation becomes gradually smaller at high frequencies. In addition, the Green's function also varies with the source position when the entropy waves are included because those waves have a much shorter wavelength than acoustic waves, and so a modest change in source position leads to different phase in the entropy distrubance incident on the nozzle. This magnifies the effect of source position on the Green's function. For this reason $\hat{G}_{1}$ must be retained inside the integral of the prediction model in Eq. (19) if the entropy waves are assumed to convect without dispersion to the nozzle. One can see from figure 7 that the rapid variation of the Green's function with frequency is strongly dependent on source position. This will be smoothed out after an integral over $\boldsymbol{y}$ for a distributed source, as illustrated in figure 8 .

\section{III.E. Paths in combustor and outer annulus}

The difference of the Green's function $\left|\hat{G}_{1}(\boldsymbol{y}, \boldsymbol{x}, \omega)\right|$ between the observer $\boldsymbol{x}$ in the combustor and outer annulus is of interest as the measured noise data were acquired in the two flow 


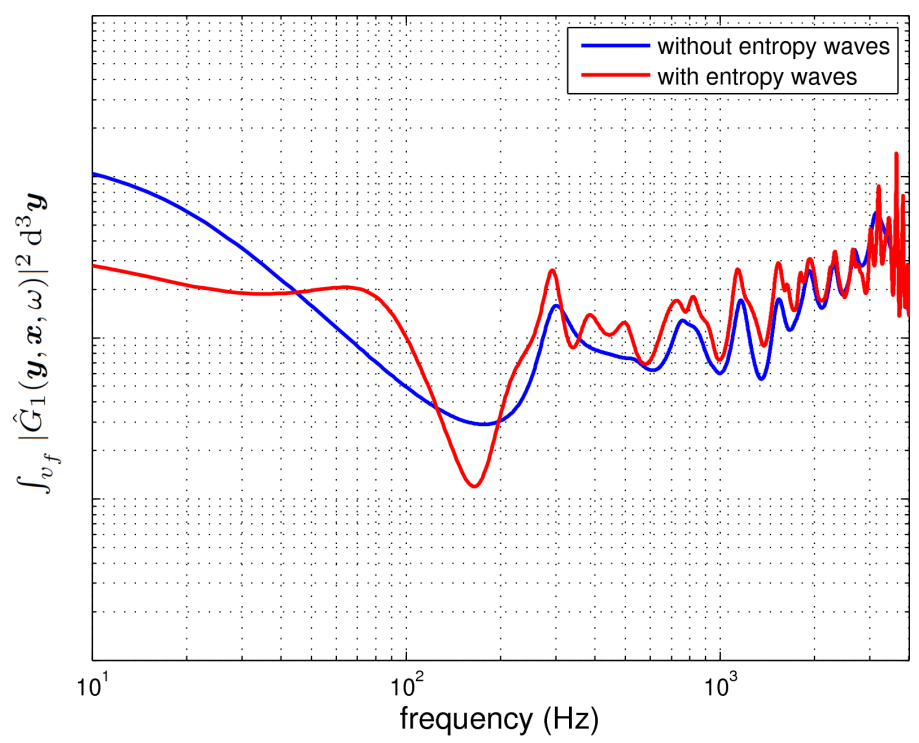

Figure 8. Effects of entropy waves on spectrum of the source integral $\int_{v_{f}}\left|\hat{G}_{1}(\boldsymbol{y}, \boldsymbol{x}, \omega)\right|^{2} \mathrm{~d}^{3} \boldsymbol{y} . \quad N=7, \theta=\pi / 3, \theta_{0}=0$. $x$ at RP 3 \& 4.

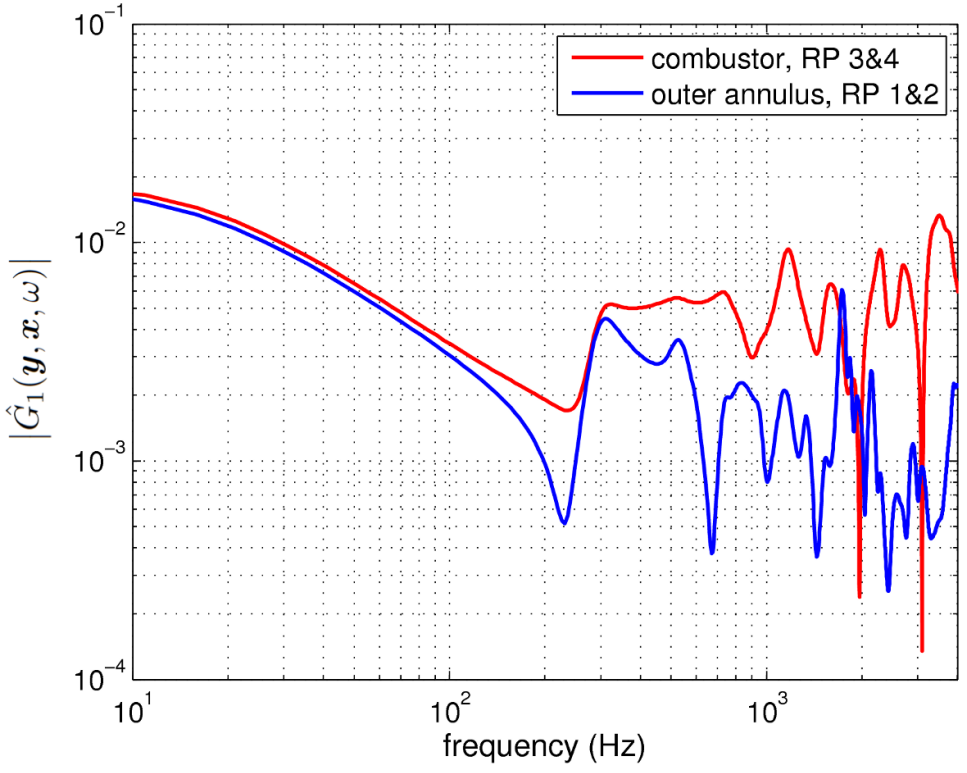

Figure 9. Comparison of spectra of the Green's function $\left|\hat{G}_{1}(\boldsymbol{y}, \boldsymbol{x}, \omega)\right|$ for observer position $\boldsymbol{x}$ in combustor (RP $3 \& 4)$ and outer annulus (RP $1 \& 2)$ ). $N=7, \theta=\pi / 3, \theta_{0}=0, x_{0}$ at combustor inlet.

paths. The spectra of $\left|\hat{G}_{1}\right|$ in the combustor (RP $3 \& 4$ ) and outer annulus (RP 1 \& 2) are compared in figure 9. It reveals that the spectral level of $\left|\hat{G}_{1}\right|$ in the combustor is above that in the outer annulus almost over the entire frequency range [10, 4000] Hz. For example, the difference at $600 \mathrm{~Hz}$ is about $4.5 \mathrm{~dB}$ in terms of SPL. From the comparison of Green's function in the two flow paths, it is straightforward to deduce that the predicted noise spectrum in the combustor would be higher than that in the outer annulus, which is consistent with experimental results as will be seen in Sec. IV. 


\section{Prediction of Combustion Noise Spectrum}

\section{IV.A. RANS data of combustor flow}

The input of mean quantities to the spectral model summarized in Sec. II.C are supplied by the results from RANS simulation of the combustor flow for the demonstrator aeroengine computed using a commercial CFD software, ANSYS FLUENT. First, the local mean flow quantities $\tilde{k}, \tilde{\epsilon}, \tilde{c}, s_{L}, a_{0}, \rho_{0}, Y_{f, 0}, \overline{\dot{w}}$ are extracted by post-processing the CFD results, and then the other quantities, $u^{\prime}, \Lambda, D a, \delta_{T}, s_{T}$, etc., are calculated from the extracted quantities. Figure 10 illustrates the predicted spatial variation of the mean reaction rate $\overline{\dot{w}}$ and fuel mass fraction $Y_{f, 0}$. It is obvious that the majority of mean reaction rate is confined within the first $15-20 \%$ of the combustor length and that the fuel mass fraction $Y_{f, 0}$ is even more concentrated towards the combustor inlet. This distribution of $\overline{\dot{w}}$ and $Y_{f, 0}$ indicates a short

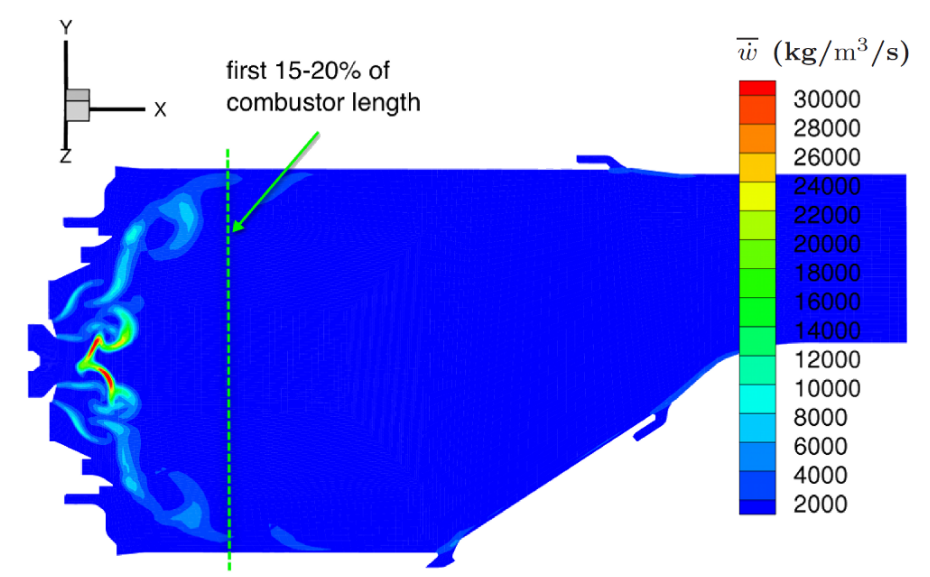

(a) mean reaction rate $\bar{w}$

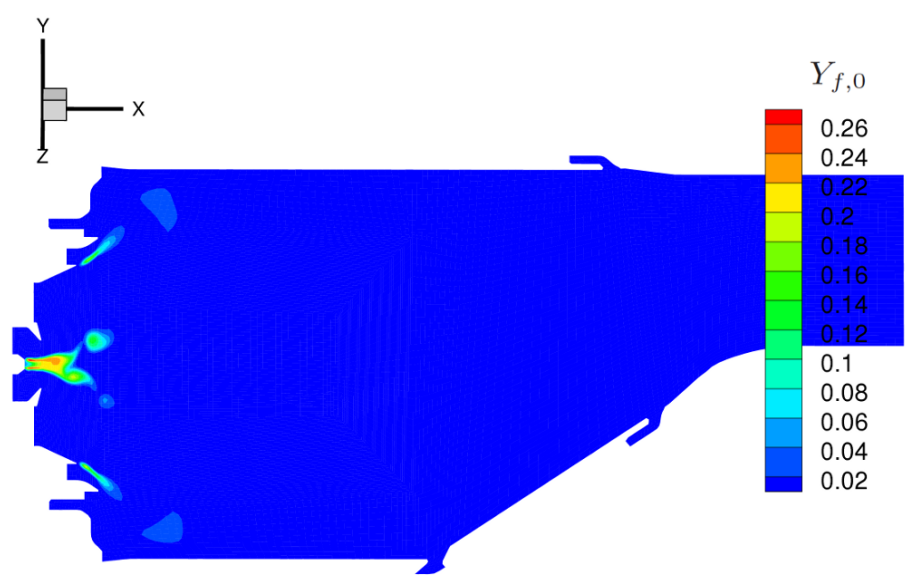

(b) fuel mass fraction $Y_{f, 0}$

Figure 10. Contours of mean reaction rate $\bar{w}\left(\mathrm{~kg} / \mathrm{m}^{3} / \mathrm{s}\right)$ and fuel mass fraction $Y_{f, 0}$ in the mid-plane of the demonstrator combustor from the FLUENT RANS data. 
flame length, and thus the processing time will be reduced significantly when the RANS data are included in the volume integral over the unsteady heat release sources. The fuel injection from the pilot and main jet can be seen in figure 10(b). For the pilot-main fuel split considered in this work, the majority of the fuel is injected via the main jet and causes most of the mean reaction rate. The relatively rich pilot mixture seen in figure $10(\mathrm{~b})$ is due to the low air flow rate in the pilot. Since rich burning is quiet, the contribution from the pilot flame to combustion noise can be ignored.

\section{IV.B. Comparison between prediction and measurement}

The prediction of combustion noise spectrum is obtained by evaluating the volume integral over the flame brush in Eq. (19). The integrand involves the Green's function and the spectral model described in Sec. II.C (see figure 1). As discussed in Sec. III.D the Green's function has different value depending on whether or not the entropy waves are assumed to have diffused before the combustor exit. Figure 11 shows the measured combustion noise spectra by the two pairs of rumble probes, together with the predictions in the combustor and outer annulus, respectively when no entropy diffusion or dispersion occurs. Only one group of data is shown for each pair of rumble probes because the differences between the spectra of the two probes are negligible for both measurements and calculations. As can be seen in figure 11, the difference in the pressure spectra between the combustor and outer annulus is captured reasonably well by the modeling method used in this study. Overall the general broadband levels are in reasonable agreement between theory and experiment.

However a number of narrowband peaks are observed in the experimental data that are not predicted in the theory. The mains hum is evident at 50 and $150 \mathrm{~Hz}$ in figure 11 on all rumble probes, particularly in the combustor. A strong combustion resonance occurs at $410 \mathrm{~Hz}$ (and its harmonics) in both flow paths and generates a narrow peak that dominates the pressure spectra, exceeding the neighboring spectral levels by some $30 \mathrm{~dB}$. This is due to self-excited thermoacoustic oscillations of the combustion system and is outside the scope of this investigation of broadband combustion noise. The frequencies of the combustion oscillations, however, can be worked out using LOTAN and they agree very well with the measured oscillation frequencies. The rumble probe measuring combustor pressure was placed at the end of the igniter tube of length $50 \mathrm{~mm}$. The measured spectra in the combustor show the expected (damped) quarter-wavelength resonance around $2500 \mathrm{~Hz}$ associated with this tube. In the prediction the pressure was calculated on the combustor wall and so there is no corresponding resonance.

In contrast, the predicted spectral shape exhibits broadband activity over the entire frequency range, with the exception of a resonance at around $1800 \mathrm{~Hz}$ in the outer annulus. The outer passage is modeled as an annulus with one end open and the other closed and 


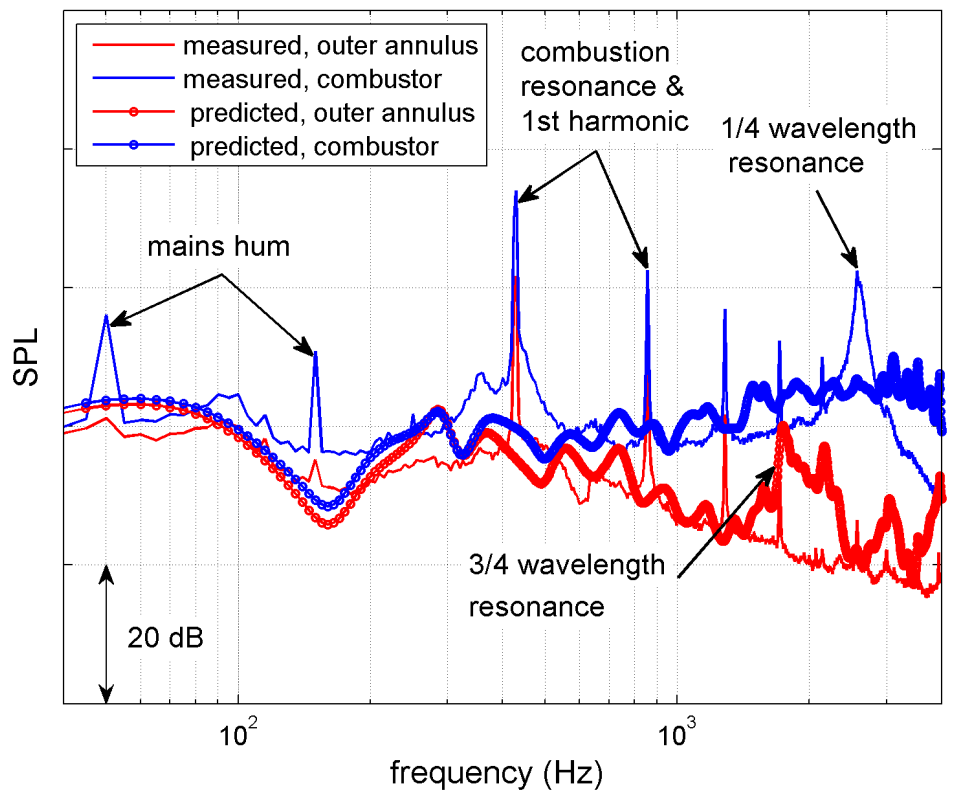

Figure 11. Comparison between predicted and measured noise spectra in the combustor and outer annulus of the demonstrator engine.

this frequency is near its 3/4-wavelength resonance. A modal analysis reveals that this peak is actually comprised of resonance frequencies of circumferential modes $[-7,7]$ that span over hundreds of Hertz. However, LOTAN may overpredict these resonance modes due to some simplifications used in the network model. For example, the downstream end of the outer annulus is not "closed" in the real engine because part of flow leaves as outer NGV (nozzle guide vane) bleed flow which would increase the damping. Nevertheless, on the whole the prediction model captures the main characteristics of the broadband spectral shape of combustion noise in the demonstrator aeroengine combustor, including the relative difference of noise spectrum between the combustor and outer annulus.

Moreover, reasonable amount of agreement in spectral level is achieved between the predicted and measured noise spectra, nearly over the entire frequency range except for very high frequencies $f \gtrsim 3000 \mathrm{~Hz}$. The difference in spectral level between the two flow paths becomes increasingly larger across this frequency range, and this is also captured in the predictions as a result of the Green's function shown in Sec. III.E. A deficiency of the spectral model ${ }^{5}$ used for the predictions has been observed in previous studies ${ }^{5,7,6}$ that it underestimates the spectral level of combustion noise at low frequencies. This deficiency, however, is not evident in figure 11 in that the comparison between predictions and measurements is reasonably good in the low frequency range, which could be attributed to the correction for the spectrum of heat release rate as described in Sec. II.C. 


\section{Conclusions}

In this paper, a theoretical model has been developed to predict the spectrum of combustion noise generated in an aeroengine combustor, and has been compared with measured noise data from a demonstrator aeroengine. The combustion noise sources are modeled using a spectral model of heat release rate by Hirsch et al., ${ }^{5}$ which has shown good quantitative agreement with recent experimental results. ${ }^{5,7,6}$ The time-averaged combustor flow of the demonstrator aeroengine has been computed using the commercial software FLUENT following RANS methodology. The CFD results indicate that the predominant combustion occurs close to the combustor inlet. This significantly reduces the computational burden for combustion noise calculation by reducing the size of the integral volume.

The computer program LOTAN has been used to calculate the transfer functions relating the linear waves, i.e. acoustic, entropic and vortical, to the heat release fluctuation. Then the Green's function was obtained by summing up the transfer functions of different circumferential modes in a thin annular combustor. When all entropy waves are assumed to be diffused before the combustor exit, the spectral content of the Green's function $\hat{G}_{1}(\boldsymbol{y}, \boldsymbol{x}, \omega)$ is observed to be insensitive to the axial source position. This saves the computational time for LOTAN and the numerical source integral. The effect of retaining the entropy waves

without dispersion or diffusion until combustor exit is to produce a variation for $\hat{G}_{1}(\omega)$ on top of the underlying trend in the spectral level. The Green's function shows some dependence on source position when the entropy waves are included at the combustor exit, but this dependence becomes very weak after an integral over the source volume. The analysis suggests a difference of about $4.5 \mathrm{~dB}$ at $600 \mathrm{~Hz}$ in the spectral level of $\hat{G}_{1}$ between the combustor and the outer annulus.

All ingredients, i.e. the Green's function determined by LOTAN, the mean reaction rate and flow quantities from the FLUENT simulation, and the spectral model, ${ }^{5}$ are required to prediction of combustion noise. The comparison between the predicted and measured noise spectra for a demonstrator aeroengine has shown that the main characteristics of the broadband spectral shape of combustion noise at a low-medium power setting is captured by the modeling framework developed in this study. The predicted spectral levels also show reasonable agreement with the measurements nearly over the entire frequency range, which indicates that the deficiency of underpredictions at low frequencies ${ }^{5,7,6}$ of the source spectral model has been ameliorated by the corrections to the model.

This work is an explorative application of the earlier source spectral model ${ }^{5}$ to the prediction of combustion noise of a realistic aeroengine combustor. The results show scope for improvement and further development. One promising avenue is to model the combustion noise sources directly through investigating the spatio-temporal correlation of heat release 
rate fluctuations in the combustor using unsteady CFD simulations or experiments, rather than translating the wave number spectrum to frequency spectrum as in the spectral model used in this paper. A direct measurement of this spatio-temporal correlation in open turbulent flames would be helpful in this regard. In addition, more flow conditions (e.g. higher power settings) and the acoustic field in the jet pipe by including the propagation of linear waves through turbine blade rows will be investigated in future work.

\section{Acknowledgments}

The current research has been conducted under UK Technology Strategy Board contract TP11/HVM/6/I/AB201K. The Noise measurements of the demonstrator aeroengine were undertaken by the German Aerospace Center (DLR). Dr. Simon Stow of Rolls-Royce plc is acknowledged for his kind assistance to the LOTAN code, and we thank Dr. Christoph Hirsch of Technische Universität München for the helpful discussions on the spectral model.

\section{References}

${ }^{1}$ Crighton, D. G., Dowling, A. P., Ffowcs Williams, J. E., Heckl, M., and Leppington, F. G., Modern Methods in Analytical Acoustics: Lecture Notes, Springer-Verlag, London, 1992.

${ }^{2}$ Rajaram, R. and Lieuwen, T., "Acoustic Radiation from Turbulent Premixed Flames," Journal of Fluid Mechanics, Vol. 637, 2009, pp. 357-385.

${ }^{3}$ Swaminathan, N., Balachandran, R., Xu, G., and Dowling, A. P., "On the Correlation of Heat Release Rate in Turbulent Premixed Flames," Proceedings of the Combustion Institute, Vol. 33, 2011, pp. 1533-1541.

${ }^{4}$ Swaminathan, N., Xu, G., Dowling, A. P., and Balachandran, R., "Heat Release Rate Correlation and Combustion Noise in Premixed Flames," Journal of Fluid Mechanics, Vol. 681, 2011, pp. 80-115.

${ }^{5}$ Hirsch, C., Wäsle, J., Winkler, A., and Sattlemayer, T., "A Spectral Model for the Sound Pressure from Turbulent Premixed Combustion," Proceedings of the Combustion Institute, Vol. 31, 2007, pp. 1435-1441.

${ }^{6}$ Wäsle, J., Winkler, A., Weyermann, F., Hirsch, C., and Sattlemayer, T., "A Model for Turbulent Combustion Noise," Acta Acoustica, Vol. 95, 2009, pp. 391-401.

${ }^{7}$ Hirsch, C., Winkler, A., Wäsle, J., and Sattlemayer, T., "Calculating the Turbulent Noise Source of Premixed Swirl Flames from Time Mean Reactive RANS Variables," The 13th International Congress on Sound and Vibration, 2006.

${ }^{8}$ Marble, F. E. and Candel, S. M., "Acoustic disturbances from gas non-uniformities convected through a nozzle," Journal of Sound and Vibration, Vol. 55, No. 2, 1977, pp. 225-243.

${ }^{9}$ Leyko, M., Moreau, S., Nicoud, F., and Poinsot, T., "Numerical and analytical modelling of entropy noise in a supersonic nozzle with a shock," Journal of Sound and Vibration, Vol. 330, No. 16, 2011, pp. 3944-3958.

${ }^{10}$ Bake, F., Richter, C., Mühlbauerc, B., Kings, N., Röhlea, I., Thiele, F., and Noll, B., "The Entropy Wave Generator (EWG): A Reference Case on Entropy Noise," Journal of Sound and Vibration, Vol. 326, No. 3-5, 2009, pp. 574-598. 
${ }^{11}$ Stow, S. R., Dowling, A. P., and Hynes, T. P., "Reflection of Circumferential Modes in a Choked Nozzle," Journal of Fluid Mechanics, Vol. 467, 2002, pp. 215-239.

${ }^{12}$ Wäsle, J. G., Vorhersage der Lärmemission Turbulenter Vormischflammen, Ph.D. thesis, Lehrstuhl Thermodynamik, Technische Universität München, 2007.

${ }^{13}$ Stow, S. R. and Dowling, A. P., "Thermoacoustic Oscillations in an Annular Combustor," ASME Paper, No. 2001-GT-0037, New Orleans, LA, USA, 2001.

${ }^{14}$ Stow, S. R. and Dowling, A. P., "Modelling of Circumferential Modal Coupling due to Helmholtz Resonantors," ASME Paper, No. GT2003-38168, Atlanta, GA, USA, 2003.

${ }^{15}$ Stow, S. R. and Dowling, A. P., "Low-Order Modelling of Thermoacoustic Limit Cycles," ASME Paper, No. GT2004-54245, Vienna, Austria, 2004.

${ }^{16}$ Dowling, A. P. and Stow, S. R., "Acoustic Analysis of Gas Turbine Combustors," Journal of Propulstion and Power, Vol. 19, No. 5, 2003, pp. 751-764.

${ }^{17}$ Hedge, U. G., Reuter, D., and Zinn, B. T., "Sound Generation by Ducted Flames," AIAA Journal, Vol. 26, No. 5, 1988, pp. 532-537.

${ }^{18}$ Goldstein, M. E., Aeroacoustics, McGraw-Hill Inc., USA, 1976.

${ }^{19}$ Spalding, D. B., "Mixing and chemical reaction in steady confined turbulent flames," 13th Symposium (International) on Combustion, 1971, pp. 649-657.

${ }^{20}$ Veynante, D. and Vervisch, L., "Turbulent Combustion modeling," Progress in Energy and Combustion Science, Vol. 28, No. 3, 2002, pp. 193-266.

${ }^{21}$ Peters, N., Turbulent Combustion, Cambridge University Press, Cambridge, UK, 2000.

${ }^{22}$ Swaminathan, N. and Bray, K. N. C., Turbulent Premixed Flames, Cambridge University Press, Cambridge, UK, 2011.

${ }^{23}$ Tennekes, H. and Lumley, J. L., A First Course in Turbulence, The MIT Press, USA, 1972.

${ }^{24}$ Kariuki, J., Dawson, J. R., and Mastorakos, E., "Measurements in turbulent premixed bluff body flames close to blow-off," Combustion and Flame, Vol. 159, No. 8, 2012, pp. 2589-2607.

${ }^{25}$ Schmid, H. P., Habisreuther, P., and Leuckel, W., "A Model for Calculating Heat Release in Premixed Turbulent Flames," Combustion and Flame, Vol. 113, 1998, pp. 79-91.

${ }^{26}$ Clavin, P. and Siggia, E. D., "Turbulent premixed flames and sound generation," Combustion Science and Technology, Vol. 78, 1991, pp. 147-155.

${ }^{27}$ Schmid, H. P., Ein Verbrennungsmodell zur Beschreibung der Wärmefreisetzung von vorgemischten turbulenten Flammen, Ph.D. thesis, Engler-Bunte-Institut, Universität Karlsruhe (TH), 1995.

${ }^{28}$ Wäsle, J., Winkler, A., and Sattlemayer, T., "Spatial Coherence of the Heat Release Fluctuations in Turbulent Jet and Swirl Flames," Flow, Turbulence and Combustion, Vol. 75, 2005, pp. 29-50. 